\title{
Immune Reconstitution Inflammatory Syndrome in children
}

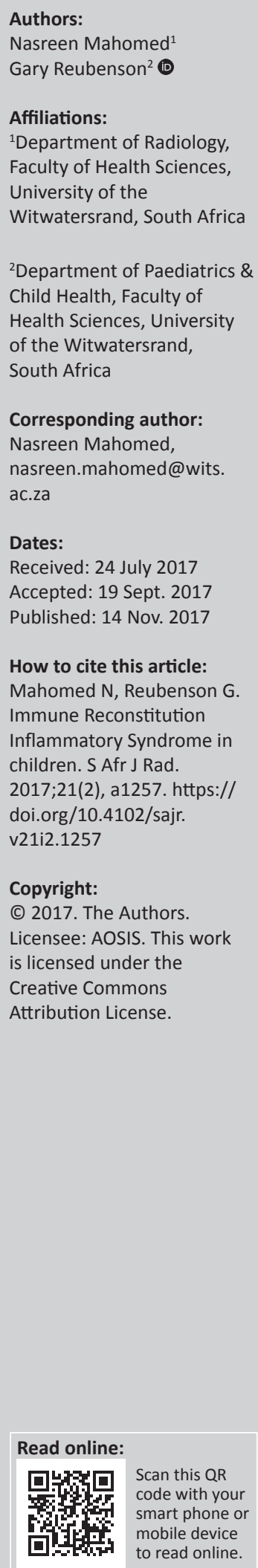

Immune Reconstitution Inflammatory Syndrome (IRIS) refers to a collection of inflammatory disorders, predominantly related to infectious processes that manifest after the initiation of antiretroviral therapy (ART) and can be classified as unmasking or paradoxical. The prevalence of IRIS in children in sub-Saharan Africa is low. Approximately half of all cases are associated with Mycobacterium tuberculosis. It may be difficult to distinguish IRIS from tuberculosis and other opportunistic infections radiologically; therefore, radiological findings must be interpreted with clinical and laboratory findings. In this review article, we describe the clinical and radiological manifestations of IRIS in children and provide illustrative radiological examples.

\section{Introduction}

Antiretroviral therapy (ART) has revolutionised the care of HIV-infected children. Compared to untreated children, life expectancy and quality of life are substantially better for children receiving ART. ${ }^{1,2}$ However, like any medication, ART has side effects and potential adverse consequences; the Immune Reconstitution Inflammatory Syndrome (IRIS) is one such adverse consequence., ${ }^{3,4,5}$

IRIS refers to a collection of inflammatory disorders, predominantly related to infectious processes that manifest after initiation of ART. It is usually a self-limiting condition, but on occasion may be severe and rarely can be life-threatening. In most cases, ART can be continued and appropriate antimicrobial therapy is provided for the relevant opportunistic infection. ${ }^{6}$

The immunological changes that occur following initiation of ART are complex, ${ }^{7,8}$ but as the name implies, IRIS occurs when ART-associated improved immune function results in an excessive immune response. ${ }^{9}$ Interestingly, paradoxical worsening of symptoms following initiation of treatment has been described in HIV-uninfected patients and follows a similar improvement in immune function. Paradoxical tuberculosis (TB) reactions are the best known examples of this. ${ }^{10,11}$

\section{Epidemiology}

Early, retrospective studies estimated IRIS incidence as high as 30\%; however, more recent evidence suggests a much lower rate of $<10 \%{ }^{5,6,12,13}$ A recent South African study using the International Network for the Study of HIV-associated IRIS criteria reported a paradoxical TBIRIS incidence of $4.8 \%$ among 104 children under 8 years of age. ${ }^{14}$ The incidence of IRIS varies according to the associated micro-organism or underlying condition, the definition of IRIS used, including whether paradoxical or unmasking forms of IRIS or both are included, the region of the world and local incidence of particular opportunistic infections and age of the children. Epidemiological studies on the overall incidence of IRIS and on specific forms of IRIS in children are limited in number and as a result of the factors mentioned above.

\section{Classification}

IRIS is typically classified as either unmasking or paradoxical; however, this distinction may be difficult, as both forms may coexist in the same child and other explanations for the findings should be considered. ${ }^{15}$

Unmasking IRIS refers to clinical features related to a previously undiagnosed, asymptomatic or subclinical infection, whereas paradoxical IRIS is worsening of a previously diagnosed or partially treated condition. Non-infectious forms of IRIS include autoimmune processes and malignancies. ${ }^{14,15,16,17}$

Precise diagnostic criteria for IRIS have been suggested, but consensus on such criteria has not been reached. However, IRIS should be strongly considered when: ${ }^{9,18}$ 
- ART has been recently initiated, usually within the last 2-6 weeks, but IRIS has been reported many months after initiation.

- There has been a substantial decline in HIV viral load, usually with a significant rise in CD4 count - however, IRIS may occur in the absence of either or both of these.

- Local or systemic inflammatory changes occur related to a previously diagnosed (paradoxical IRIS) or unrecognised (unmasking IRIS) infectious condition - however, IRIS has been reported for non-infectious conditions, including malignancies and autoimmune diseases.

- An alternative explanation for these changes is not identified. This can be very challenging as progression of HIV, new opportunistic infections, drug-resistant pathogens, and medication side effects may present similarly.

\section{Risk factors}

The risk for and severity of IRIS are largely informed by two factors. Firstly, the degree of CD4+ T-cell immune suppression; the lower the pre-treatment CD4 count, the higher the risk of IRIS. ${ }^{13}$ Secondly, the virological and immunological responses to ART; the more rapid the decline in HIV viral load and the faster the rise in CD4 count, the greater the likelihood of developing IRIS. ${ }^{13,18}$ This likely explains the declining incidence in paediatric IRIS as children are being diagnosed earlier and are started on ART soon thereafter, usually before progression to profound immune suppression. ${ }^{19}$

\section{Clinical manifestations}

Clinical manifestations of IRIS are dependent on the implicated pathogen and frequently involve worsening of previously identified manifestations or the onset of new symptoms, similar to those encountered with non-IRISrelated infections with the same pathogen.

Mycobacterium tuberculosis (TB) is implicated in approximately half of adults with IRIS. ${ }^{21}$ TB is less commonly implicated in paediatric IRIS, possibly related to the paucibacillary nature of paediatric TB. ${ }^{14}$ Other pathogens commonly implicated are Bacille Calmette-Guérin (BCG) vaccine, ${ }^{22,23}$ cytomegalovirus $(\mathrm{CMV})^{24}$ and non-tuberculous mycobacteria (NTM) ${ }^{25}$ Less common causes of paediatric IRIS include herpes simplex, Kaposi sarcoma (caused by human herpesvirus 8), ${ }^{26,27}$ Cryptococcus neoformans, ${ }^{28}$ Pneumocystis jiroveci $i^{29}$ and parvovirus B19. ${ }^{30}$ This differs from adults, where cryptococcal IRIS ${ }^{28}$ is more likely and BCG-IRIS essentially does not occur. Systemic manifestations include fever, weight loss, fatigue and pain..$^{20}$ Common clinical manifestations of IRIS in children are tabulated in Table 1. In children dermatological manifestations such as seborrhoeic dermatitis and herpes zoster are not uncommon, generally mild and not associated with radiological changes.

\section{Radiological manifestations}

\section{Mycobacterium tuberculosis-associated Immune Reconstitution Inflammatory Syndrome}

Radiologically it is important to distinguish IRIS from TB or other opportunistic infections, including NTM and CMV.
Drug-resistant TB is a clinical and laboratory diagnosis, not a radiological diagnosis and both drug-sensitive and drugresistant TB can manifest IRIS reactions. ${ }^{17,31}$

The most common radiological manifestations of TB-IRIS occur within the chest and include new or worsening hilar or mediastinal lymphadenopathy, which may cause tracheobronchial compression. Other common chest radiological findings include worsening or new air space consolidation, pleural effusions, reticular infiltrates or nodular infiltrates (Figures 1-3). ${ }^{14,16,17,31}$

Abdominal manifestations of TB-IRIS include ascites, abdominal lymphadenopathy, and splenic micro-abscesses. ${ }^{16}$ Central nervous system (CNS) manifestations are uncommon and include TB meningitis, which may cause obstructive hydrocephalus, or associated tuberculomas. ${ }^{16}$

\section{Bacille Calmette-Guérin-associated Immune Reconstitution Inflammatory Syndrome}

BCG-associated IRIS is well recognised ${ }^{16}$ and in some settings is the most commonly identified cause. ${ }^{16,22}$ It usually manifests with inflammatory changes at the injection site and/or within the ipsilateral draining lymph nodes. Chest radiographic changes are uncommon and may be indistinguishable from TB. ${ }^{22}$ Unusual manifestations of BCGIRIS have been reported (Figure 4).

\section{Mycobacterium avium complex Immune Reconstitution Inflammatory Syndrome}

Mycobacterium avium complex (MAC)-associated IRIS manifests radiologically as necrotic lymph nodes identified on ultrasound, computed tomography or magnetic resonance imaging. These may be indistinguishable from other mycobacterial infections. The diagnosis of MAC may be made from tissue culture; ${ }^{16}$ however, cultures are commonly negative. Therefore, a high level of clinical suspicion is required.

TABLE 1: Common clinical manifestations of Immune Reconstitution Inflammatory Syndrome.

\begin{tabular}{|c|c|c|}
\hline Pathogen & Clinical features & Comments \\
\hline TB & $\begin{array}{l}\text { - Worsening respiratory complaints } \\
\text { - Symptoms related to airway } \\
\text { compression } \\
\text { - Pleural effusion (new or } \\
\text { progression) } \\
\text { - Lymphadenitis } \\
\text { - Central nervous system } \\
\text { involvement, including focal } \\
\text { signs, seizures and meningism }\end{array}$ & $\begin{array}{l}\text { - New central nervous } \\
\text { system lesions may be } \\
\text { asymptomatic } \\
\text { - Aspirates from lymph } \\
\text { nodes commonly } \\
\text { culture-negative }\end{array}$ \\
\hline BCG & $\begin{array}{l}\text { - Inflammatory changes and } \\
\text { abscess formation at injection } \\
\text { site or draining lymph nodes } \\
\text { (axillary and cervical) } \\
\text { - Rarely, evidence of } \\
\text { disseminated disease (e.g. } \\
\text { hepatosplenomegaly or } \\
\text { osteomyelitis) }\end{array}$ & $\begin{array}{l}\text { - Localised disease is } \\
\text { generally a clinical } \\
\text { diagnosis that seldom } \\
\text { requires microbiological } \\
\text { confirmation }\end{array}$ \\
\hline $\mathrm{CMV}$ & $\begin{array}{l}\text { - Pneumonitis } \\
\text { - Retinitis }\end{array}$ & $\begin{array}{l}\text { - Pneumonitis more } \\
\text { common than in adults, } \\
\text { but retinitis much less } \\
\text { common }\end{array}$ \\
\hline $\begin{array}{l}\text { NTM (e.g. } \\
\text { Mycobacterium } \\
\text { avium) }\end{array}$ & $\begin{array}{l}\text { - Lymphadenitis } \\
\text { - Abdominal complaints }\end{array}$ & $\begin{array}{l}\text { - Frequently culture- } \\
\text { negative with well-formed } \\
\text { granulomas on histology }\end{array}$ \\
\hline
\end{tabular}



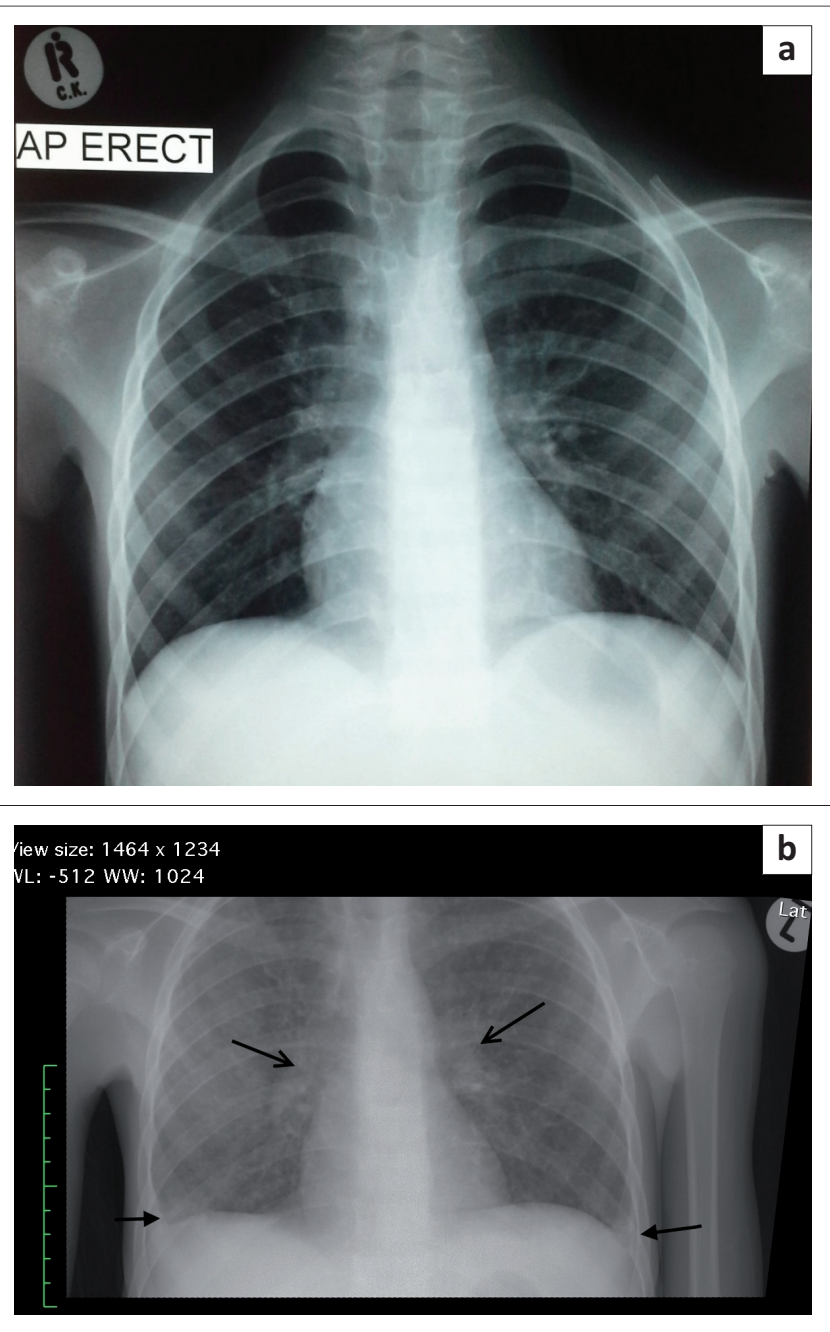

ART, antiretroviral therapy; IRIS, immune reconstitution inflammatory syndrome; TB, tuberculosis.

FIGURE 1: An 11-year-old HIV-infected boy, CD4 count 9 cells $/ \mathrm{mm}^{3}(4 \%)$, was initiated on ART. He developed progressive symptoms consistent with disseminated TB and responded well to continuation of ART and standard fourdrug TB treatment. Frontal chest radiograph taken at the time of ART initiation (a) demonstrates a normal chest radiograph. Frontal chest radiograph (b) taken 3 weeks after ART initiation demonstrates a diffuse reticular nodular infiltrate with bilateral hilar lymphadenopathy (open arrows) and small pleural effusions (closed arrows). A diagnosis of unmasking TB-IRIS was made.

Radiologically, it may be difficult to distinguish IRIS from TB and other opportunistic infections so radiological findings must be interpreted in combination with clinical and laboratory findings. ${ }^{16,17,31}$

\section{Non-radiological investigation}

Investigation is largely informed by the particular infection under consideration. Measurement of viral load and CD4 counts are helpful, particularly when poor adherence to therapy is suspected. A significant decline in viral load from baseline, as well as a substantial increase in CD4 count, is suggestive of IRIS, but not essential for making the diagnosis. ${ }^{918}$ Of note, children with suspected local or regional BCG-IRIS can generally be diagnosed based on clinical manifestations and microbiological confirmation using lymph node biopsy or fine-needle aspiration is seldom warranted unless alternative diagnoses are under consideration. If
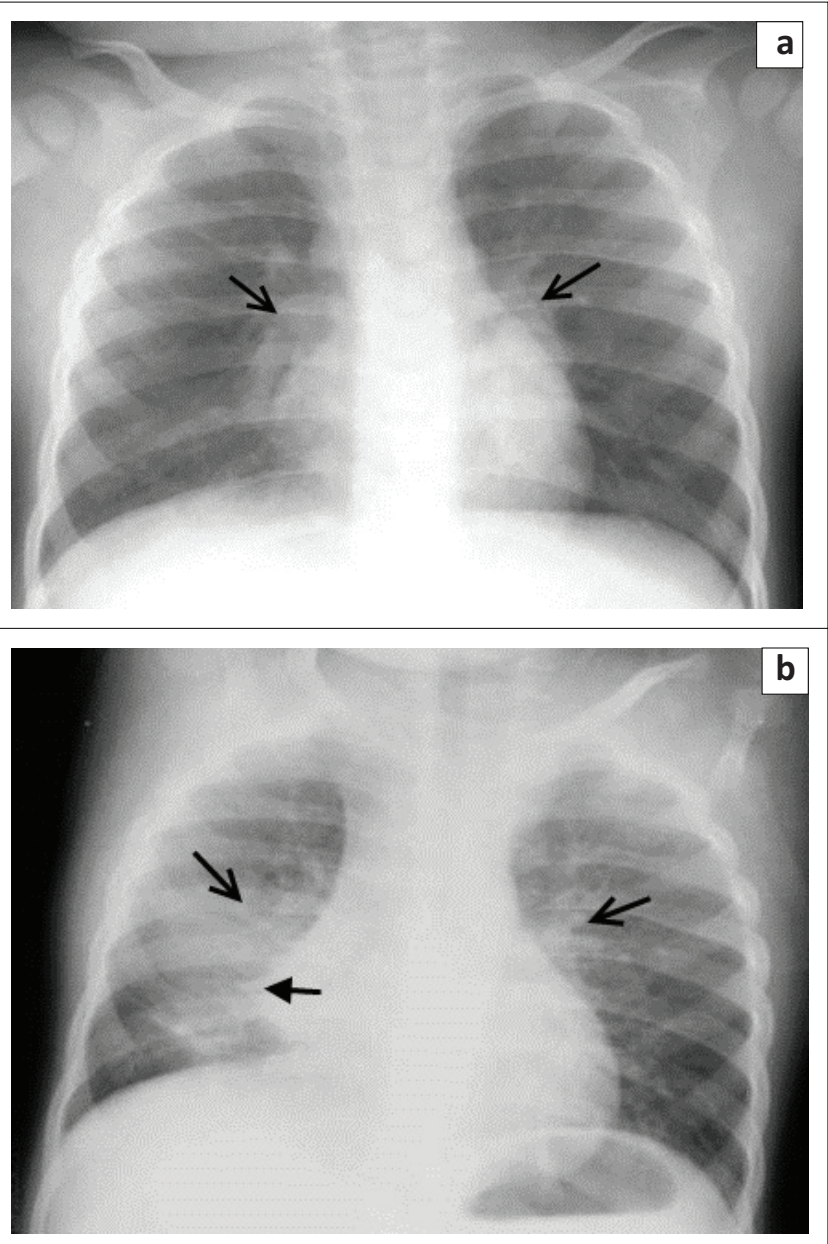

ART, antiretroviral therapy; IRIS, immune reconstitution inflammatory syndrome; TB, tuberculosis.

FIGURE 2: A 3-year-old HIV-infected girl initiated on ART 2 weeks after starting TB treatment. CD4 count was not available. Frontal chest radiograph taken at the time of ART initiation: (a) demonstrates bilateral hilar lymphadenopathy (open arrows). Chest radiograph taken 2 weeks after ART initiation; (b) demonstrates right middle lobe consolidation and collapse (closed arrow), with worsening hilar lymphadenopathy (open arrows). In combination with the clinical, radiological and laboratory findings, a diagnosis of paradoxical TB-IRIS was made.

samples are submitted from patients with suspected BCGrelated disease, it is vital to indicate this on the laboratory request form so that appropriate testing to differentiate TB from BCG can be performed.

\section{Clinical management}

The most important components of effective management are to treat the IRIS-related infection using the same modalities as for non-IRIS-related infections with that pathogen. Because IRIS is frequently mild and self-limiting, in most patients continuation of ART is to be encouraged. ${ }^{6}$ In patients with severe or lifethreatening IRIS, including airway compression by hilar or mediastinal lymph nodes or expansile CNS mass lesions, it may rarely be appropriate to interrupt ART until their condition stabilises, whereafter ART can be safely restarted. ${ }^{6}$ Stopping ART is not to be taken lightly and when being considered should involve discussion with a specialist or subspecialist.

In children with pain and discomfort from BCG-IRIS lymphadenitis, consideration should be given to needle 

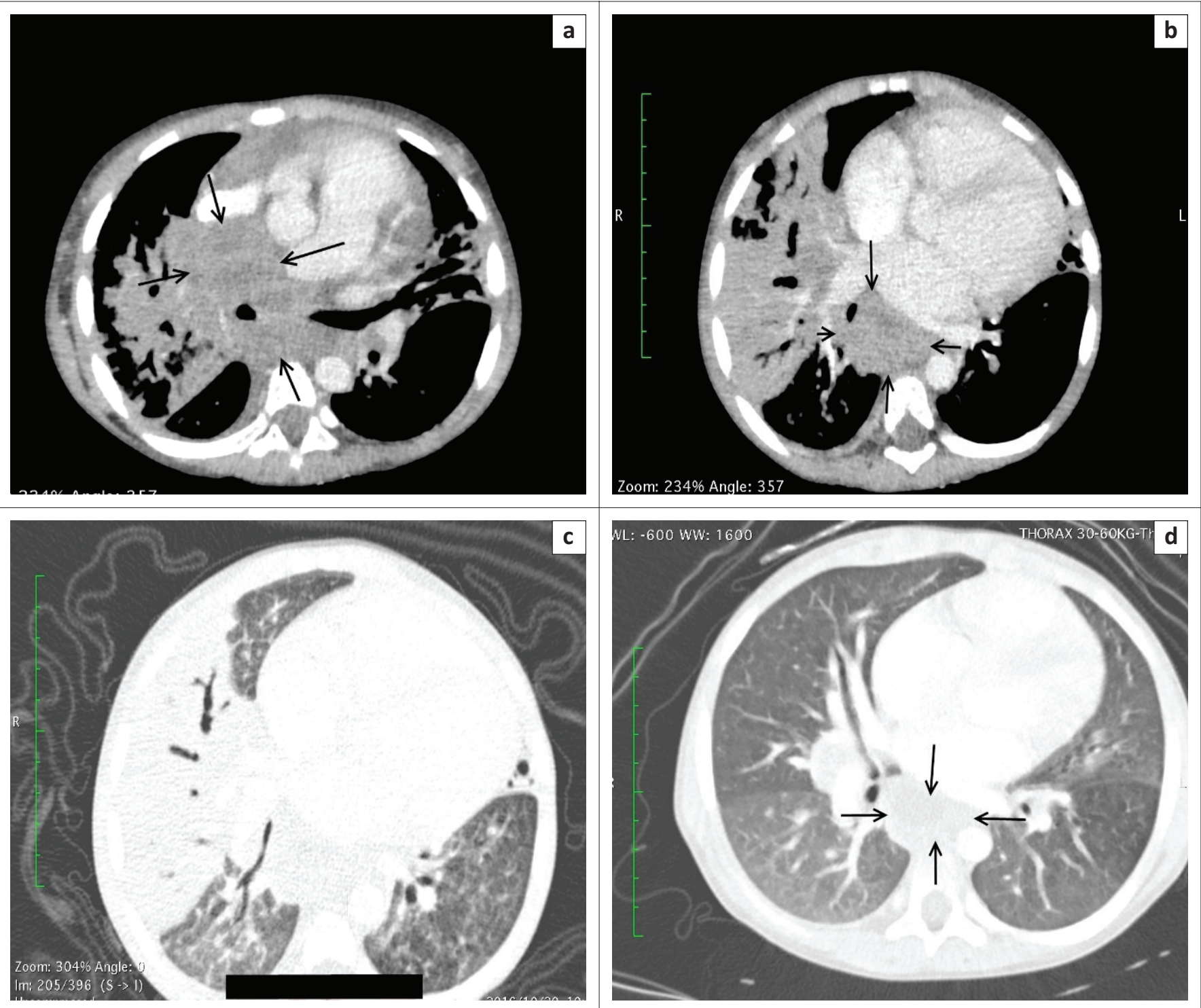

ART, antiretroviral therapy; CT, computed tomography; IRIS, immune reconstitution inflammatory syndrome; TB, tuberculosis.

FIGURE 3: A 4-year-old HIV-infected boy living with an adult diagnosed with pulmonary TB was initiated on ART. Three months later, he developed symptoms of pulmonary TB, which was microbiologically confirmed (Unmasking IRIS). He was initiated on appropriate TB treatment and initially improved; 3 weeks later he presented with fever and worsening respiratory symptoms and signs (Paradoxical IRIS). Computed tomography of the chest, axial, soft tissue window (a and b) demonstrates mediastinal and hilar lymph nodes (arrows) encasing the bronchi bilaterally. There is also posterior mediastinal involvement with anterior displacement of the left atrium and a small right pleural effusion; (c) axial lung window demonstrates dense right middle lobe and apical segment of right lower lobe consolidation with lingular consolidation. A diagnosis of paradoxical and unmasking TB-IRIS was made. ART and TB treatment were continued and corticosteroids were administered; he demonstrated a good clinical response and is currently well; (d) axial CT chest axial lung window performed 6 months later demonstrates resolution of the consolidation, with residual lymphadenopathy (open arrows).

aspiration of fluctuant areas to relieve symptoms. Such aspiration may need to be repeated. ${ }^{22}$ In addition to continuation of ART and treating the implicated pathogen, patients with IRIS may benefit from anti-inflammatory medicines. In mild and moderate cases, non-steroidal anti-inflammatory drugs (NSAIDs) have been used to provide symptomatic relief and may hasten IRIS resolution. In more severe cases, corticosteroids may be required for a number of weeks, whereafter they can be tapered and stopped. ${ }^{6}$ The additional immune suppression related to corticosteroid use is a concern in such patients and specialist input is advised before their commencement.

\section{Prevention}

If HIV is diagnosed early and ART initiated soon thereafter, IRIS is unlikely. ${ }^{23}$ Recent improvements in early infant diagnosis of HIV followed by almost immediate initiation of ART have resulted in IRIS becoming uncommon. ${ }^{23}$

Furthermore, it is important to consider opportunistic infection prior to ART initiation as initiating treatment for them first may reduce the risk of IRIS developing. This is particularly true for CNS TB and cryptococcal meningitis where ART initiation should be delayed during the initial weeks of treatment in order to reduce the risk of IRIS. Tuberculous mass lesions (tuberculomas) have been associated with life-threatening CNS-IRIS. ${ }^{13,32}$ In most patients with TB, ART can be safely initiated about 2 weeks after TB treatment has commenced; however, extrapolating from experience in adults with HIV-associated cryptococcal meningitis, ART initiation should be delayed by at least $6-8$ weeks. ${ }^{33}$ Routine prophylactic use of NSAIDs or corticosteroids to reduce the 

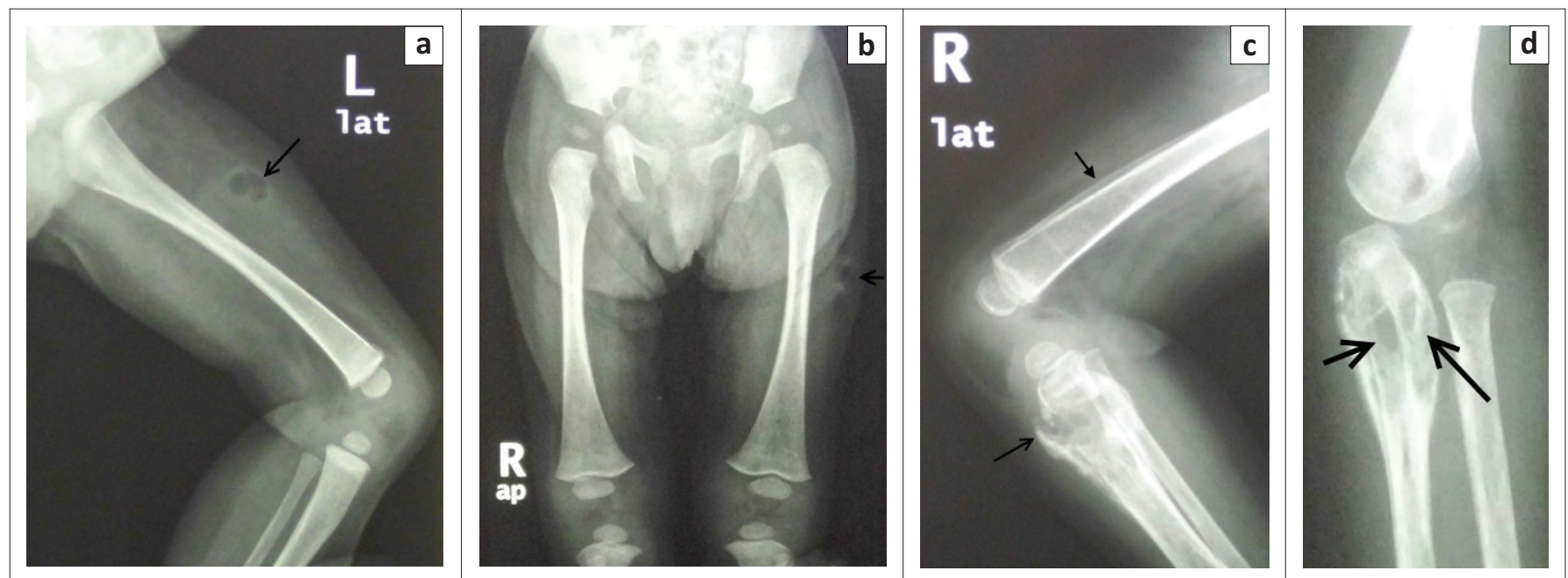

ART, antiretroviral therapy; BCG, Bacille Calmette-Guérin; IRIS, immune reconstitution inflammatory syndrome.

FIGURE 4: A 9-month-old HIV-infected girl, with CD4 count 9 cells $/ \mathrm{mm}^{3}(1.53 \%)$, was admitted with severe malnutrition, right axillary and left thigh abscesses, generalised lymphadenopathy, hepatosplenomegaly and clinical evidence of HIV encephalopathy. ART was initiated with subsequent worsening of the thigh abscess. An aspiration was performed on the left thigh abscess which cultured Mycobacterium bovis BCG, confirmed using molecular testing. Appropriate antimycobacterial treatment was provided: (a) (lateral) and (b) (AP), radiograph of the lower limbs demonstrate an air containing abscess in the upper left lateral thigh soft tissues (open arrow) and generalised left lower limb soft tissue swelling. No periosteal reaction was demonstrated in visualised bones. She was readmitted a year later, still on antimycobacterial treatment with soft tissue abscesses in the right thigh, forehead, abdominal wall and left forearm; (c) (left lateral knee) demonstrates osteomyelitis, with lytic lesions within the proximal tibial metaphysis and a cortical break along the anterosuperior tibial margin (open arrow). There is periosteal reaction along the tibia and distal femur (closed arrow) with generalised soft tissue oedema; (d) (left AP forearm) demonstrates another focus of osteomyelitis with lytic lesions in the proximal metadiaphysis of the ulna (open arrows), associated periosteal reaction and osteopenia of the left ulna and radius. Bone aspiration again confirmed $M$. bovis BCG, now resistant to first-line antimycobacterial agents, necessitating surgical drainage; however, the patient did not survive. A diagnosis of BCG-IRIS was made.

risk of IRIS is not recommended, rather patients at high risk for IRIS should be closely monitored for new or worsening symptoms so that IRIS can be timeously identified and treated.

\section{Conclusion}

While ART has revolutionised the care of HIV-infected children, ART has side effects and potential adverse consequences which include IRIS. IRIS is typically classified as either unmasking or paradoxical; however, this distinction may be difficult, as both forms may coexist in the same child and other explanations for the clinical findings should be considered. Approximately half of all IRIS (adult and paediatric) cases are associated with M. tuberculosis. The commonest radiological manifestations of TB-IRIS include new or worsening hilar or mediastinal lymphadenopathy, worsening or new air space consolidation, pleural effusions, reticular infiltrates or nodular infiltrates. It may be difficult radiologically to distinguish IRIS in children from TB and other opportunistic infections so radiological findings must be interpreted in combination with clinical and laboratory findings.

\section{Acknowledgements}

We would like to acknowledge Dr Gillian Sorour for her contribution towards the clinical cases.

\section{Competing interests}

The authors declare that they have no financial or personal relationship(s) which may have inappropriately influenced them in writing this article.

\section{Authors' contributions}

Both authors contributed equally to writing the article, thus co-first authors. G.R. was predominantly responsible for the clinical component and N.M. for the radiology.

\section{References}

1. Nesheim SR, Kapogiannis BG, Soe MM, et al. Trends in opportunistic infections in the pre- and post-highly active antiretroviral therapy eras among HIV-infected children in the Perinatal AIDS collaborative transmission study, 1986-2004. Pediatrics. 2007;120(1):100-109. https://doi.org/10.1542/peds.2006-2052

2. Kourtis AP, Bansil P, Posner SF, et al. Trends in hospitalizations of HIV-infected children and adolescents in the United States: Analysis of data from the 19942003 Nationwide Inpatient Sample. Pediatrics. 2007;120(2):e236-e243. https:// doi.org/10.1542/peds.2006-3268

3. Michelet C, Arvieux C, Francois C, et al. Opportunistic infections occurring during highly active antiretroviral treatment. AIDS. 1998;12(14):1815-1822. https://doi. org/10.1097/00002030-199814000-00013

4. Hirsch $\mathrm{HH}$, Kaufmann $\mathrm{G}$, Sendi $\mathrm{P}$, et al. Immune reconstitution in HIV-infected patients. Clin Infect Dis. 2004;38(8):1159-1166. https://doi.org/10.1086/383034

5. Shelburne SA, Montes M, Hamill RJ. Immune reconstitution inflammatory syndrome: More answers, more questions. J Antimicrob Chemother. 2006;57(2):167-170. https://doi.org/10.1093/jac/dki444

6. Murdoch DM, Venter WD, Feldman C, et al. Incidence and risk factors for the immune reconstitution inflammatory syndrome in HIV patients in South Africa: A prospective study. AIDS. 2008;22(5):601-610. https://doi.org/10.1097/QAD.0b013e3282f4a607

7. Pakker NG, Notermans DW, de Boer RJ, et al. Biphasic kinetics of peripheral blood $T$ cells after triple combination therapy in HIV-1 infection: A composite of redistribution and proliferation. Nat Med. 1998;4(2):208-214. https://doi. org/10.1038/nm0298-208

8. Autran B, Carcelain G, Li TS, et al. Positive effects of combined antiretroviral therapy on CD4+ T cell homeostasis and function in advanced HIV disease. Science. 1997;277(5322):112-116. https://doi.org/10.1126/science.277.5322.112

9. Breton G, Duval X, Estellat C, et al. Determinants of immune reconstitution inflammatory syndrome in HIV type 1-infected patients with tuberculosis after initiation of antiretroviral therapy. Clin Infect Dis. 2004;39(11):1709-1712. https://doi.org/10.1086/425742

10. Smith $\mathrm{H}$. Paradoxical responses during the chemotherapy of tuberculosis. J Infect. 1987;15(1):1-3. https://doi.org/10.1016/S0163-4453(87)91276-X

11. Chambers ST, Hendrickse WA, Record C, et al. Paradoxical expansion of intracranial tuberculomas during chemotherapy. Lancet. 1984;2(8396):181-184. https://doi. org/10.1016/S0140-6736(84)90478-1 
12. Ratnam I, Chiu C, Kandala NB, et al. Incidence and risk factors for immune reconstitution inflammatory syndrome in an ethnically diverse HIV type 1-infected cohort. Clin Infect Dis. 2006;42(3):418-427. https://doi.org/10.1086/499356

13. Grant PM, Komarow L, Andersen J, et al. Risk factor analyses for immune reconstitution inflammatory syndrome in a randomized study of early vs. deferre org/10.1371/journal.pone.0011416

14. Van Rie A, Sawry S, Link-Gelles R, et al. Paradoxical tuberculosis-associated immune reconstitution inflammatory syndrome in children. Pediatr Pulmonol 2016;51(2):157-164. https://doi.org/10.1002/ppul.23221

15. Haddow LJ, Easterbrook PJ, Mosam A, et al. Defining immune reconstitution inflammatory syndrome: Evaluation of expert opinion versus 2 case definitions in a South African cohort. Clin Infect Dis. 2009;49(9):1424-1432. https://doi. org/10.1086/630208

16. Kilborn T, Zampoli M. Immune reconstitution inflammatory syndrome after initiating highly active antiretroviral therapy in HIV-infected children. Pediat Radiol. 2009;39(6):569-574. https://doi.org/10.1007/s00247-009-1192-y

17. Zampoli M, Kilborn T, Eley B. Tuberculosis during early antiretroviral-induced immune reconstitution in HIV-infected children. Int J Tuberc Lung Dis. 2007;11(4):417-423.

18. Manabe YC, Campbell JD, Sydnor E, et al. Immune reconstitution inflammatory syndrome: Risk factors and treatment implications. J Acquir Immune Defic Syndr. 2007;46(4):456-462. https://doi.org/10.1097/QAl.0b013e3181594c8c

19. Penazzato $M$, Prendergast AJ, Muhe LM, et al. Optimisation of antiretroviral therapy in HIV-infected children under 3 years of age. Cochrane Database Syst Rev. 2014(5):CD004772. https://doi.org/10.1002/14651858.CD004772.pub4

20. Cheng VC, Yuen KY, Chan WM, et al. Immunorestitution disease involving the innate and adaptive response. Clin Infect Dis. 2000;30(6):882-892. https://doi. org/10.1086/313809

21. Narita M, Ashkin D, Hollender ES, et al. Paradoxical worsening of tuberculosis following antiretroviral therapy in patients with AIDS. Am J Respir Crit Care Med. 1998;158(1):157-161. https://doi.org/10.1164/ajrccm.158.1.9712001

22. Smith K, Kuhn L, Coovadia A, et al. Immune reconstitution inflammatory syndrome among HIV-infected South African infants initiating antiretroviral therapy. AIDS 2009;23(9):1097-1107. https://doi.org/10.1097/QAD.0b013e32832afefc
23. Rabie $\mathrm{H}$, Violari A, Duong $\mathrm{T}$, et al. Early antiretroviral treatment reduces risk of bacille Calmette-Guerin immune reconstitution adenitis. Int I Tuberc Lung Dis. 2011;15(9):1194-1200. https://doi.org/10.5588/ijtld.10.0721

24. Miller RF, Shaw PJ, Williams IG. Immune reconstitution CMV pneumonitis. Sex Transm Infect. 2000;76(1):60. https://doi.org/10.1136/sti.76.1.60

25. Phillips P, Bonner S, Gataric N, et al. Nontuberculous mycobacterial immune reconstitution syndrome in HIV-infected patients: Spectrum of disease and long-term follow-up. Clin Infect Dis. 2005;41(10):1483-1497. https://doi.org/10.1086/497269

26. Tobian AA, Grabowski MK, Serwadda D, et al. Reactivation of herpes simplex virus type 2 after initiation of antiretroviral therapy. J Infect Dis. 2013;208(5):839-846. https://doi.org/10.1093/infdis/jit252

27. Bower $M$, Nelson $M$, Young $A M$, et al. Immune reconstitution inflammatory syndrome associated with Kaposi's sarcoma. J Clin Oncol. 2005;23(22):52245228. https://doi.org/10.1200/JCO.2005.14.597

28. Sungkanuparph S, Jongwutiwes $U$, Kiertiburanakul S. Timing of cryptococcal immune reconstitution inflammatory syndrome after antiretroviral therapy in patients with AIDS and cryptococcal meningitis. J Acquir Immune Defic Syndr. 2007;45(5):595-596. https://doi.org/10.1097/QAl.0b013e318061b5eb

29. Barry SM, Lipman MC, Deery AR, et al. Immune reconstitution pneumonitis following Pneumocystis carinii pneumonia in HIV-infected subjects. HIV Med. 2002;3(3):207-211. https://doi.org/10.1046/j.1468-1293.2002.00115.x

30. Watanabe $\mathrm{D}$, Taniguchi $\mathrm{T}$, Otani $\mathrm{N}$, et al. Immune reconstitution to parvovirus $\mathrm{B} 19$ and resolution of anemia in a patient treated with highly active antiretroviral therapy. Infect Chemother. 2011;17(2):283-287. https://doi.org/10.1007/s10156-010-0111-3

31. Theron S, Andronikou S, George R, et al. Non-infective pulmonary disease in HIVpositive children. Pediatr Radiol. 2009;39(6):555-564. https://doi.org/10.1007/ s00247-009-1156-2

32. Zolopa A, Andersen J, Powderly W, et al. Early antiretroviral therapy reduces AIDS progression/death in individuals with acute opportunistic infections: A multicente randomized strategy trial. PLoS One. 2009;4(5):e5575. https://doi.org/10.1371/ journal.pone.0005575

33. Bisson GP, Molefi M, Bellamy S, et al. Early versus delayed antiretroviral therapy and cerebrospinal fluid fungal clearance in adults with HIV and cryptococcal meningitis. Clin Infect Dis. 2013;56(8):1165-1173. https://doi.org/10.1093/ $\mathrm{cid} / \mathrm{cit019}$ 